\title{
Particle Entrainment and Deposition Scenario in Sublayer Region of Variable Area Conduit
}

\author{
Esam Jassim ${ }^{1, *}$ \\ ${ }^{1}$ Department of Mechanical Engineering, Prince Mohammad Bin Fahd University, 31952 Khobar, Saudi Arabia
}

\begin{abstract}
The study presents the particle deposition and aggregation phenomena by introducing new parameter called Particle Deposition Number PDN, defined as the ratio of the particle instantaneous velocity to its capturing value. The particle capture or rebound fate will decide from knowing such number. The study employed new scheme of particle deposition in the sublayer region which includes balancing of four forces. Moreover, the bouncing model is also considered for particle fate decision. The study examines the variation of particle velocity at varying area tube and the critical velocity in which particle will tend to stick if its velocity is lower than the threshold limit. The results show that threshold velocity is exponentially decreased with the increment in the particle size. Capturing of particles is shown to be enhanced as the conduit converges due to increasing in the PDN. The analysis of the deposition also investigates the impact of the particle size on the PDN. At low flow velocity, the NDP has V-shaped trend as particle size increases. However, veering toward constant PDN value has occurred as the flow velocity augmented. Finally, small sized particles experience rebound due to the prevailing of the particle impact energy over the adhesion energy before impacting with the surface. The dissipation in the particle energy during impaction causes large sized particle to loose greater amount of energy compare to small sized one, resulting in domination of the adhesion part, which leads to deposition on the surface.
\end{abstract}

\section{Introduction}

Deposition mechanism of particles suspended in gas flow is reported as a critical concern in many petrochemical industries, power plants, and environment protection agencies. Economic and environmental concerns over coal have recently driven research towards reducing the drawbacks caused by post-combustion products, mainly fly ash. As the demand of coal fuel is boosted, the negative impaction occurred by the airborne particles evolved after combustion on the environment was and is still paid attention [1]. The morphology of the particulates, known as fly ash, has tendency to deposit and stick on the internal surfaces of the flue gas carrier. The deposition and accumulation of such undesirable particulates result in augmenting in the thermal resistance of the heat exchange surface and may also cause corrosion [2]. In worse case, particle deposition could cause complete blockage of pipes and therefore subsequently cause degrading the operating life of the system $[3,4]$. Cleaning the afflicted surface, on the other hand, is costly and time consuming [5]. Hence, modeling the deposition and aggregation processes becomes crucial to mitigate the amount of the accumulated particulates.
Particle migration and deposition phenomena have been addressed in literatures for decades. Early research extensively focused on the development of empirical correlations to accurately present the trajectory of the tiny particles. Furthermore, theoretical analysis has also been proposed to quantify the deposition rate and verify the outcome with the available experimental data. Lately in the twentieth century, the utilization of computer via individual simulation or software coding has added vast progression to the understanding of the particle behavior during deposition.

Particle Deposition rate is influenced by particle size. Models of particle deposition phenomena for different sizes have been proposed by many researchers either analytically or numerically. Study conducted by Hossain et al [6] found that tiny particles with less inertia have higher propensity to stay with the continues phase. Model to track the particles mobility near the wall developed by Longmire [7], was contain a correction term to include the particle dispersion. Li and Ahmadi [8], studied the contribution of Brownian diffusion, flow turbulence, and gravity of small particles to the deposition rate. Fan and Ahmadi [9], modeled the deposition velocity that applied to vertical smooth and rough surfaces. Ounis et al [10], numerically simulated nano and micro size particle dispersed in turbulent flow. Jassim et al incorporated the

Corresponding author: ejassim@pmu.edu.sa 
particle nucleation and deposition in orifice into CFD code.

The main parameters causing the surface to capture the particle was identified and studied by many researchers [11]. In summary, they all concluded that particles with velocity less than a critical value were captured by the surface. Measurement of Rogers and Reed led to model the particle adhesion by evaluating the elastic-plastic impaction [12].

Deposition of Particle-laden flow is influenced by the structure of the fluid-solid interaction that dominates the near-wall region in turbulent flow, [13]. The formation of the boundary layer border splits the no-slip condition region from the free stream inviscid region, causing severe changing in the map description of the forces exerted on fluid and solid particles.

The situation in the boundary layer distinct becomes even more sophisticated in the turbulent flow since the BL itself is divided into two sub-regions: the viscous sublayer and the fully turbulent layer. Both shares the limit of $\left(\mathrm{u}^{+}=\mathrm{Y}^{+}=10\right)$ evenly. In the present work, we attempt to tackle the particle behavior in the sublayer region through incorporating the major forces that eventually help in tracking the particle motion. The objective of this paper is to incorporate a new model proposed by Jassim et al [14], with other existing models and implements them on fly ash particle deposition then compare the predictions to available literatures.

\section{The philosophy of th e $p$ article deposition}

In the sublayer distinct, the particle mobility, the particle entrainment or deposition, and the surface sticking/detached are all influenced by the turbulence intensity, fluid properties, particle-wall interactions, particle inertia, gravity, lifting and drag coefficients, and the size of the particle itself.

The particle fate is decided based on the net force acting on the particles. The present study adopts the model proposed by Jassim et al [14], as illustrated in fig. (1).

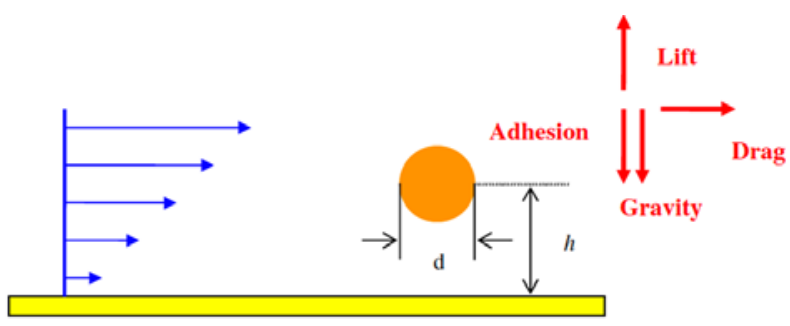

Fig. 1. Forces acting on a particle within sublayer region

Since the fly ash formed after coal combustion possesses much higher density than the continuous phase and the number of immiscible particles is low, the particle-particle collision is neglected, [5].

\section{Model of Deposition}

The particle inertia is balanced with the forces acting on the particle. The momentum per unit mass in the $x$ direction for the forces exerted on the particle immersed in sublyer region is:

$\frac{d u_{p}}{d t}=F_{D}\left(u-u_{p}\right)+g_{x}\left(1-\frac{\rho}{\rho_{p}}\right)+\sum F_{x}$

where $u$ is velocity of the continuous phase, $u_{p}$ is the velocity of the spherical particle, $\rho$ and $\rho_{p}$ are the fluid density and particle density, respectively. $\mathrm{F}_{\mathrm{D}}\left(\mathrm{u}-\mathrm{u}_{\mathrm{p}}\right)$ is the drag force per unit particle mass. $F_{x}$ is the additional forces per unit particle mass terms exerted on the particle. Rudinger [15] has sum-up the forces applied on the immiscible particles as in the following expression:

$\sum F_{\text {particle }}=$ drag force + added mass effect + history effect + gravitational force + buoyancy force + lift force + intercollision force + Brownian force + Thermophoresis force + Magnus forces +Basset Force

Description of each force has been extensively elaborated in the literatures, for instance El-Batsh et. al, [16]. However, a part from Rudinger expression, the forces illustrated in Fig. (1) are the only forces to be included in the analysis since the other forces have a little contribution to the deposition rate, as reported by many literatures, for instance $[5,16]$.

\subsection{The deposition velocity model of particle}

A new correlation merged the Brownian effect and the inertia term reported by Jassim et al [14], is employed here. It takes the form of:

$V_{d}^{+}=\frac{V_{d}}{u^{*}}=\left\{\begin{array}{l}0.2 S c^{-2 / 3} \mathrm{Re}^{-1 / 8}+\frac{1}{2}\left[\frac{\left(0.64 k^{+}+0.5 d_{p}^{+}\right)^{2}}{3.42}\right]^{1 /\left(1+\tau^{\tau^{2}} L_{1}^{+}\right)} \text {if } V_{d}^{+}<0.14 \\ \times\left[1+8 e^{-(\tau-10)^{2} / 32}\right] \frac{0.037}{1-\tau^{\tau^{2}} L_{1}^{+}} \\ 0.14\end{array}\right.$

where $L_{1}^{+}=\frac{3.08}{S d_{p}^{+}} ; S=\frac{\rho_{p}}{\rho_{f}} ; \quad d_{p}^{+}=\frac{d_{p} u^{*}}{v_{g}} \quad k^{+}=\frac{k u^{*}}{v_{g}}$

$k^{+}$is the dimensionless surface roughness ( which is zero for smooth surface)

$$
\tau^{+}=\frac{\tau u^{*}}{v}=\frac{\rho_{p} d_{p}^{2} u^{*^{2}} C_{c}}{18 \rho v^{2}}
$$

$\mathrm{u}^{*}$ is the frictional velocity which is averaged to $7 \%$ of the free stream velocity.

Cc is the Cunningham correction factor and depends on the Knudson number, which in turns is defined as the ratio between the mean free path and particle diameter. For air at ambient temperature, the mean free bath is of order of one-tenths of particle diameter. Hence, the value of Cc would be less or equal 1.12. 
Once the particle impacts the surface, it could stick to the surface or bounce back to the flow. The benchmark used to determine the fate of the particle is the Particle Capture Velocity PCV. The particle sticks to the surface if its normal velocity is lower than the PCV. Numerous models of PCV are proposed in the literatures. However, an empirical expression derived by [19] is selected to obtain the PCV $V_{s}$ :

$$
\begin{gathered}
V_{s}=\left(\frac{2 E}{d_{p}}\right)^{10 / 7} \\
E=051\left[\frac{5 \pi^{2}\left(k_{1}+k_{2}\right)}{4 \rho_{p}^{3 / 2}}\right]^{2 / 5} k_{1}=\frac{\left(1-\zeta_{s}^{2}\right)}{\pi E_{s}} \quad k_{2}=\frac{\left(1-\zeta_{p}^{2}\right)}{\pi E_{p}}
\end{gathered}
$$

The time-dependent velocity of the particle can be obtained by solving the following ODE:

$$
\begin{aligned}
& \text { Assuming: } \\
& \theta=u-u_{p}
\end{aligned}
$$

Eq. 1 becomes:

$\frac{d \theta}{d t}=-F_{D} \theta-g_{x}\left(1-\frac{\rho}{\rho_{p}}\right)-0.039\left(\frac{3 \pi}{4}\right) d_{p}-1.615 \mu d_{p}^{2} \sqrt{\frac{K_{r}}{v}} \theta$

\section{Rearranging:}

$\frac{d \theta}{d t}+\left(F_{D}+1.615\left(\frac{\mu d_{p}^{2}}{m_{p}}\right) \sqrt{\frac{K_{r}}{v}}\right) \theta=-g_{x}\left(1-\frac{\rho}{\rho_{p}}\right)-0.039\left(\frac{3 \pi}{4}\right)\left(\frac{d_{p}}{m_{p}}\right)$

or: $\frac{d \theta}{d t}+\left(C_{1}+C_{2}\right) \theta=-\left(C_{3}+C_{4}\right)$

where:

$$
\begin{aligned}
& C_{1}=\left(\frac{3}{4}\right)\left(\frac{\mu C_{D} \operatorname{Re}_{p}}{\rho_{p} d_{p}^{2}}\right) ; C_{2}=\left(\frac{9.69}{\pi}\right)\left(\frac{\rho}{\rho_{p}}\right)\left(\frac{u^{*}}{d_{p}}\right) ; \\
& C_{3}=g_{x}\left(1-\frac{\rho}{\rho_{p}}\right) ; C_{4}=\left(\frac{9 W_{A}}{2 \rho_{p} d_{p}^{2}}\right)
\end{aligned}
$$

Solving the ODE by applying the $\mathrm{BC}$ that at $\mathrm{t}=0$, the particle locates at the border of the sublayer with a velocity of $\boldsymbol{V}_{\boldsymbol{d}}$ leads to:

$$
\frac{u_{p}}{u}=1-\left\{\left(1-\frac{V_{d}}{u}\right) e^{-\left(C_{1}+C_{2}\right)}+\left(\frac{C_{3}+C_{4}}{C_{1}+C_{2}}\right)\left(\frac{e^{-\left(C_{1}+C_{2}\right)}-1}{u}\right)\right\}
$$

\section{Rebound phenomena}

When the ash particle possesses a velocity less than the threshold capturing value, it would be captured by the surface. However, rebounce could also be resulting after particle-surface intraction. The decision on whether the particle sticks or rebounds depends on the domination of the adhesion energy or the incoming particle kintetic energy, as seen in the fig. (2).

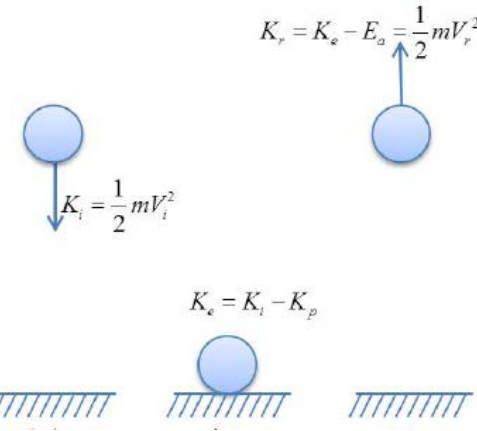

Fig. 2. particle bounce process as a simple energy balance for normal impacts, [2]

Bitter determined the energy dissipation as a result of particle plastic deformation from the following expression [20]:

$$
\begin{gathered}
K_{p}=\left[\left(k_{i}-\frac{k_{y}}{16}\right)^{1 / 2}-\left(\frac{15 k_{y}}{16}\right)^{1 / 2}\right]^{2} k_{y}=\frac{1}{2} m_{p} V_{s}^{2} ; \\
k_{i}=\frac{\left(1-\zeta_{p}^{2}\right)}{\pi E_{p}}
\end{gathered}
$$

Rogers and Reed model is employed to determine the condition of particle bouncing after interacting with a surface. The particle attempts to rebound for the following

$$
k_{i}-K_{p} \geq E_{a} \quad \text { or } \quad \psi=\frac{k_{i}-K_{p}}{E_{a}} \geq 1
$$

condition:

$E_{a}$ is the total adhesion energy at the particle-surface contact; $\psi$ is the bouncing factor.

\section{Results and discussion}

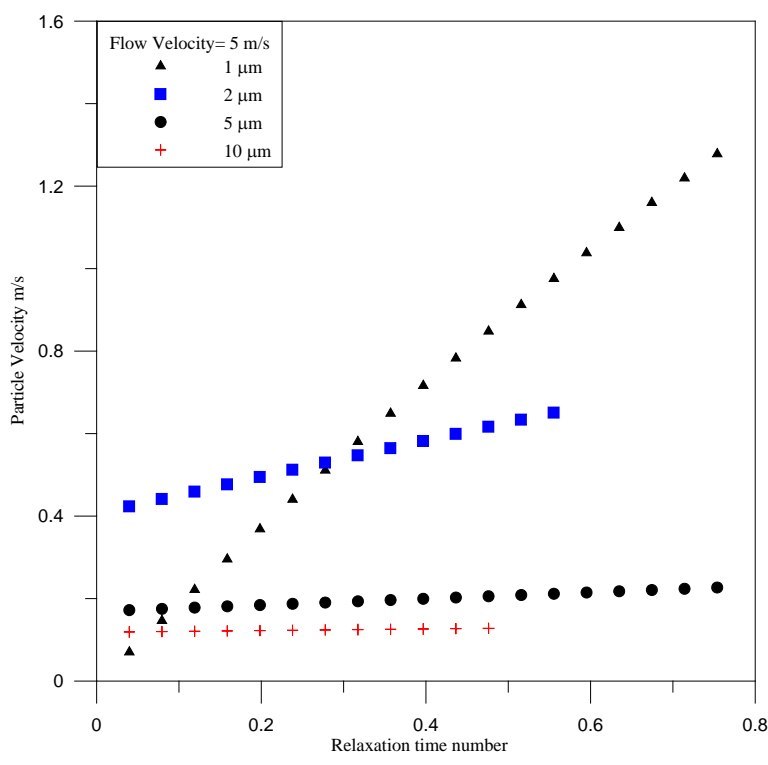

Fig. 3 particle velocity in the sublayer zone at different velocities of the continuous phase 
Fig (3) presents the particle velocity variation in the sublayer region at relatively low inlet flow velocity. Tiny particles have less tendency to deposit as the velocity they possessed is more likely to overlap the capturing limit. Larger particles, however, tend to deposit and stick on the surface, which agreed what Hussain et al founded.

The threshold velocity at different particle size is evaluated and sketched in fig. (4). It is shown that the critical velocity below which the particle captured by the surface, is exponentially dropped as the particle becomes larger. The conclusion agrees the outcome obtained by Dong et al. [2].

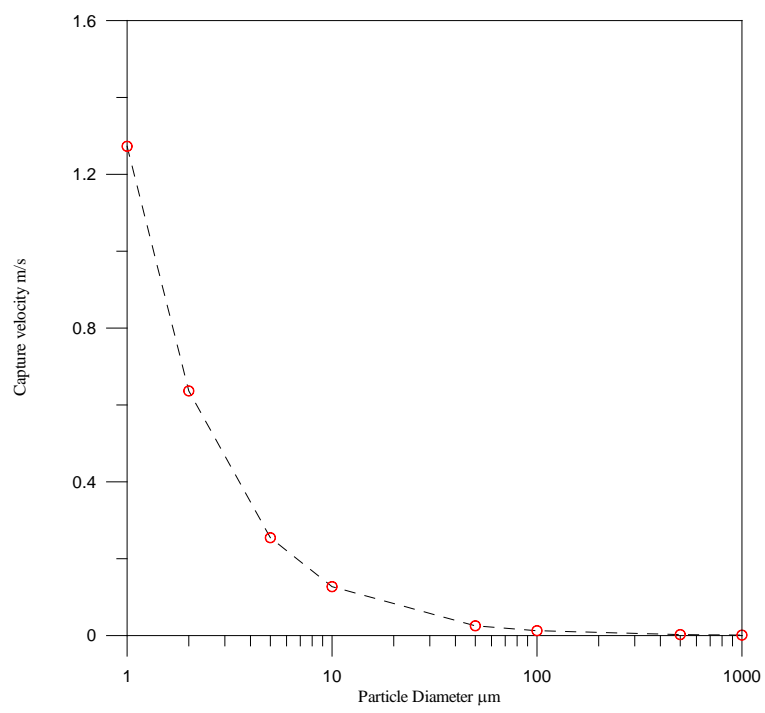

Fig. 4. the threshold limit below which particle will be captured

\subsection{Introduction the deposition velocity number}

A dimensionless parameter is defined to describe the sticking vs floating behavior of particle in the sublayer region. Deposition Velocity Number (DVN) is defined as the ratio of the particle instantaneous velocity to its capturing limit.

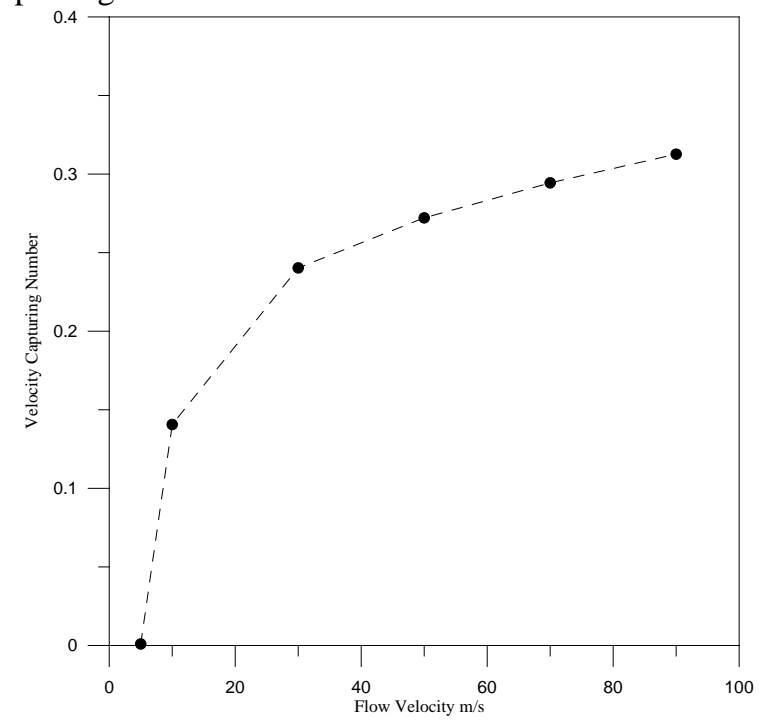

Fig. 5. Deposition Velocity Number (DVN) variation with flow velocity
Fig (5) depicts the influence of inlet flow velocity on the capturing velocity. By using the velocity capturing number as a benchmark, it is clearly illustrated that the flow velocity does impact the deposition of the particle. However, the trend is non-linear rather polynomialrelated function.

The interesting conclusion obtained from fig (5) is that at high flow velocity, capturing is more likely to improve. CFD simulation provides identical conclusion as seen in fig. (6), which presents the concertation of deposition particles along the variable area conduit. As expected, the concentration of the deposited particles increases with the area of the conduit decreases, reaching the peak limit at the throat since the highest flow velocity is at this position.

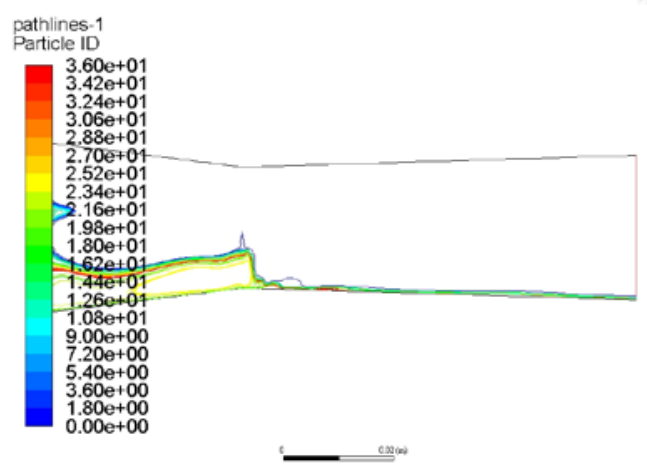

Fig. 6. deposition concentration near the surface along varying area conduit

The impact of flow velocity on the capturing number is sketched in figure (7). The figure is generated based on the assumption suggested by Fan and Ahmadi that the deposition velocity in the turbulent flow is constant [9]. It is obvious that at high flow speed the particle size has insignificant impact on the capturing process in the sublayer region. This attributes to the fact that the incident velocity at the boundary layer border plays the important role in the capturing and sticking process. However, at low flow speed, the size of particle has significant rule in the deposition due to the gravity to drag ratio which is high for low speed flow.

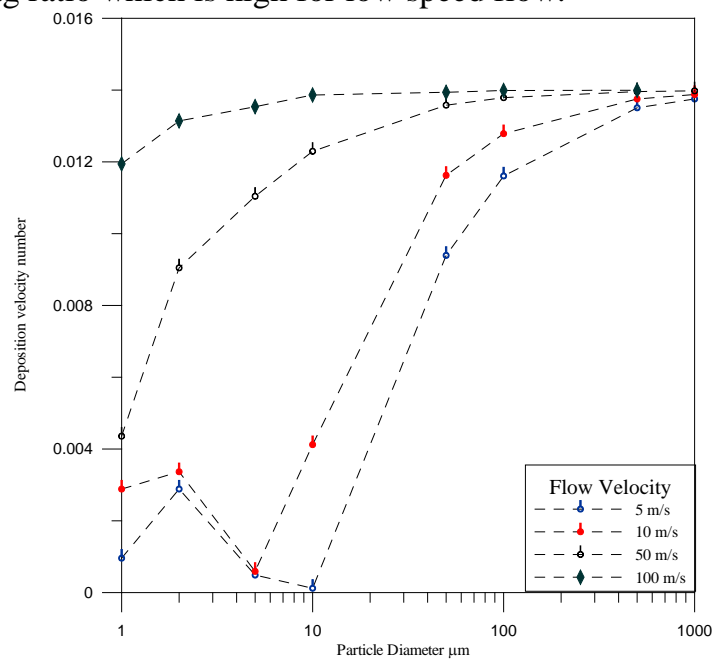

Fig. 7. Deposition Velocity Number (DVN) for different particle sizes 


\subsection{Inspection of rebounding}

Particle fate is decided by the bouncing factor stated in eq. 9. Figure 8 presents the bouncing factor for different particle size. The figure shows that the rebound process is sensitive for small particles at low fluid velocity. Large particle are shown to be less sensitive making them experience final capture once they impact the surface. The result is in consistence with Dong et al. and Liu et al. $[2,21]$, who introduced that energy dissipated, was the prominent factor in the behavior of small-sized particles.

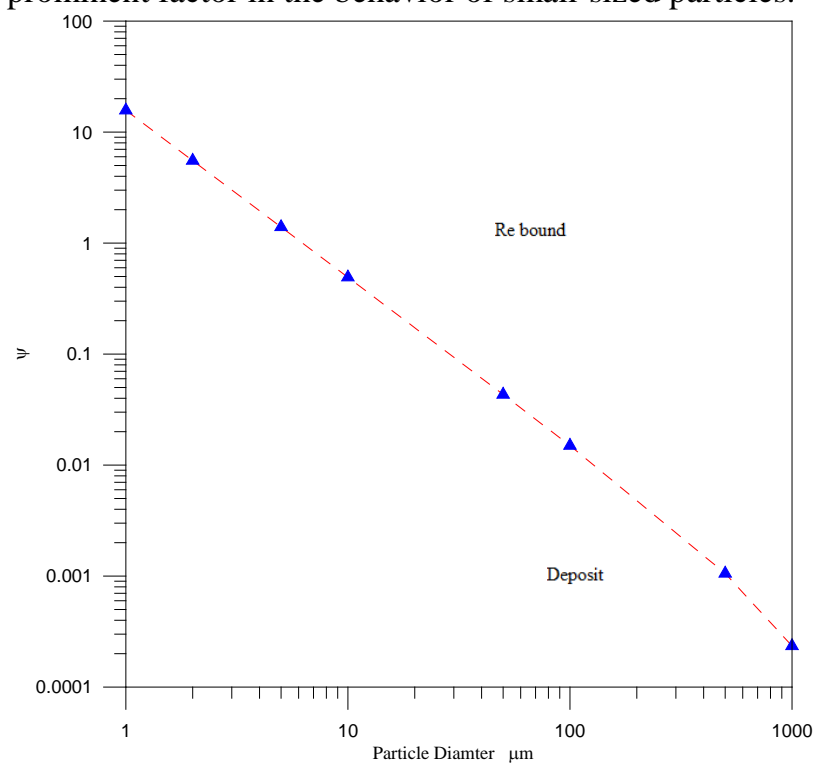

Fig. 8 Particle fate in the sublayer region

\section{Conclusion}

Although the demand of coal is ongoing for at least next couple decades, the negative impaction of the postcombustion products could limit the utilization of the coal-operated systems. The resultant ash whether deposits on the heat transfer surfaces or mobiles in the environment could curb the feasibility of such systems. The deposition process of particles flowing in a variable area conduit that is subjected to various forces in the sublayer region is studied. The capture/rebound fate of the particle is determined by the net force exerted on the particle and the its velocity relative to the threshold limit. The study has been conducted on particles with sizes ranging from $1-1000 \mu \mathrm{m}$. CFD simulation is employed to support the conclusion of the analysis. The following summarizes the study outcome:

- Particle concentration increases at conduit section with high flow velocity.

- Large particles have greater inclination to deposit on the surface of the conduit than small particles due to the inertia they possess.

- The threshold velocity capturing limit is inversely proportionate to the particle size.

- The deposition velocity number, on the other hand, is influenced significantly by the flow velocity for small sized particles.
- Unlike large sized particles that have a slim chance of rebounding, results show that small-sized particles experience bouncing due to the high kinetic energy they possess.

\section{References}

1. Esam I. Jassim, "CFD Modeling of Toxic Element Evolved during Coal Combustion”, Arabian Journal for Science and Engineering: Volume 40, Issue 12 (2015), Page 3665-3674.

2. M. Dong, S. Li, J. Xie, J. Han. Experimental studies on the normal impact of fly ash particles with planar surfaces. Energies, 6 (7) (2013), pp. 3245-3262.

3. Jassim E., Abedinzadegan Abdi M., and Muzychka Y., "Computational Fluid Dynamics Study for Flow of Natural Gas through High Pressure Supersonic Nozzles: Part 1- Real Gas Effects and Shockwave”, Journal of Petroleum Science and Technology, Vol. 26, issue 15, 1757-1772, 2008.

4. Jassim E., Abedinzadegan Abdi M., and Muzychka Y., "Computational Fluid Dynamics Study for Flow of Natural Gas through High Pressure Supersonic Nozzles: Part 2- Nozzle Geometry and Vorticity”, Journal of Petroleum Science and Technology, Vol. 26, issue (15), 1773-1785, 2008.

5. B. J. Barker, - Simulation of Coal Ash Deposition on Modern Turbine Nozzle Guide Vanes, thesis, The Ohio State University, 2010.

6. Hossain, A., and J. Naser. "CFD Investigation of Particle Deposition around Bend Ina Turbulent Flow." 15th Australasian Fluid Mechanics Conference (2004)

7. Longmire, Pamela. "Computational Fluid Dynamics (CFD) Simulations of Aerosol in a U-Shaped Steam Generator Tube." Thesis. Texas A\&M University, 2007.

8. Li, A. and Ahmadi, G., "Dispersion and Deposition of Spherical Particles from Point Sources in a Turbulent Channel Flow”, Aerosol Science and Technology, 16:209-226, 1992.

9. Fan, F-G. and Ahmadi, G. (1993). A sublayer model for turbulent deposition of particles in vertical ducts with smooth and rough surfaces. Journal of Aerosol Science, 24, 45-64

10. Ounis, H.; Ahmadi, G. and Mclaughlin, J.B. (1991), Dispersion and Deposition of Brownian Particles from Point Sources in a Simulated Turbulent Channel Flow, Journal of Colloid and Interface Science, Vol. 147, No.1, pp. 233-250.

11. Nielsen, H.P.; Baxter, L.L.; Sclippab, G.; Morey, C.; Frandsen, F.J.; Dam-Johansen, K. Deposition of potassium salts on heat transfer surfaces in strawfired boilers: A pilot-scale study. Fuel 2000, 79, 131-139. 
12. Rogers, L.N.; Reed, J. The adhesion of particles undergoing an elastic-plastic impact with a surface. J. Phys. D Appl. Phys. 1984, 17, 677-689.

13. Jassim, E.I. (2018), 'Geometrical Impaction of supersonic nozzle on the dehumidification performance during Gas purification process experimental study', Arabian Journal for Science and Engineering, https://doi.org/10.1007/s13369-0183340-X

14. Esam I. Jassim, Abedinzadegan Abdi M., and Muzychka Y., "A new approach to investigate hydrate deposition in gas-dominated flowlines", Journal of Natural Gas Science and Engineering, Vol.2, Issue 4, Sept.2010, Pages 163-177.

15. Rudinger, G., "Fundamentals of gas-particle flow", Elsevier Scientific Pub. Co., Amsterdam, New York, 1980.

16. El-Batsh, Hersham. "Modeling Particle Deposition on Compressor and Turbine Blade Surfaces." Thesis. Vienna University of Technology, 2001.
17. Saffman, P. G. (1965), The Lift on a Small Sphere in a Slow Shear Flow, Journal of Fluid Mechanics, Vol. 22, Part 2, pp. 385-400.

18. Kallio GA, Reeks MW. 1989. A numerical simulation of particle deposition in turbulent boundary layers. Int. J. Multiph. Flow 15:433-46.

19. El-Batsh, H. and H. Haselbacher, "Numerical Investigation of the Effect of Ash Particle Deposition on the Flow Field through Turbine Cascades," IGTI, Amsterdam, Netherlands, GT-2002-30600 (2002).

20. Bitter, J.G.A. A study of erosion phenomena part I. Wear 1963, 6, 5-21.

21. Liu, G.; Li, S.; Yao, Q. A JKR-based dynamic model for the impact of micro-particle with a flat surface. Powder Technol. 2011, 207, 215-223. 